\title{
Optimization-based Attitude Determination in Geodetic Applications
}

https://doi.org/10.1515/comp-2020-0146

Received Oct 07, 2019; accepted Apr 14, 2020

\begin{abstract}
A new approach to determining the attitude of a rigid body is suggested, which does not rely on the use of magnetometers. In the framework of this approach, the problem of determining the attitude reduces to solving a minimization problem for a function of three variables (angles characterizing the initial attitude of the body). The proposed method can be employed in precise geodetic measurements carried out with the use of a geodetic pole with a satellite antenna and an IMU installed on its top when, for some reasons, the surveyor cannot position the pole vertically (e.g., near walls or buildings). The use of the traditional approach, which relies on a compass and accelerometers, in this case does not ensure the desired accuracy of the attitude determination due to magnetic disturbances (both external ones and those induced by the receiver) affecting badly compass readings. The discussion is illustrated by results of field experiments.
\end{abstract}

Keywords: rigid body attitude, Poisson kinematic equation, 3-axis gyro, GNSS measurements

\section{Introduction}

We address the problem of estimating the attitude, or orientation, of a rigid body subjected to rotational motion. The attitude of a rigid body (device) is determined traditionally with the help of inertial measurement units (IMUs), which include gyros, accelerometers, and magnetometers (e-compass). While gyros are needed when the body is moving, the attitude of an immovable body is determined by means of accelerometers and magnetometers. The accuracy of determining the attitude depends on character-

\footnotetext{
${ }^{\star}$ Corresponding Author: Alexander Pesterev: Institute of Control Sciences, Moscow, Russia; Email: a.pesterev@javad.com Ivan Matrosov: Javad GNSS, Moscow, Russia; Email: i.matrosov@javad.com Yury Morozov: Institute of Control Sciences, Moscow, Russia; Email: y.morozov@ejavad.com
}

๑ Open Access. ( 2020 A. Pesterev et al., published by De Gruyter. (cc) BY 4.0 License istics of the sensors used and, mainly, on the unaccounted biases of the sensors, which can vary in time and depend on the temperature. Because of distortions of Earth's magnetic field caused by both external magnetic anomalies and magnetic fields induced by currents in the measurement units, accuracy of compass measurements is often insufficient for determining the attitude precisely. It should also be noted that, whereas biases of gyros can easily be determined in the course of a quite simple calibration procedure immediately before taking measurements, determining biases of accelerometers and, especially, magnetometers requires a more complicated calibration procedure $[1,2]$. The calibration of magnetometers becomes much more involved when currents in the measurement units vary in the course of body motion, with the calibration parameters being time-averaged quantities (see, e.g., [3]), which results in very inaccurate compass measurements.

A specific feature of the case of a moving body is that the accelerometers measure apparent acceleration (the sum of the linear acceleration of the body and the gravity vector) rather than the acceleration of gravity. The problem here is that, unlike the gravity vector, the apparent acceleration vector is not known in the inertial frame. To address this problem, various observers have been developed that fuse IMU measurements with the body velocities (measured using GPS), see, e.g., [4], and references therein.

In this paper, we suggest a new approach to determining the attitude of a moving body with a fixed point that does not use accelerometers and magnetometers. To determine the attitude matrix of a device equipped with a GNSS receiver, satellite antenna, and a three-axis gyro, we use measurements of the angular velocity vector and the linear velocity of the antenna.

Precise attitude determination is needed in a great number of various applications. For example, in land surveying, to determine the position of a point on the ground, the bottom end of a geodetic pole with a satellite antenna $A$ on its upper end (Figure 1) is placed at the point to be measured (point $O$ in Figure 1) and the pole is fixed in a vertical position (using a bubble level) for some time. Then, the coordinates of the point $O$ in the plane of the local horizon 
are taken to be those of the antenna (to be more specific, of the phase center of the antenna), and the vertical coordinate is taken to be that of the antenna minus the length of the pole. In certain circumstances, the pole cannot be positioned vertically. When the pole is inclined, the position of the antenna can be recalculated into that of the bottom end with the help of the attitude matrix, which is usually determined by the inclinometer and compass measurements. The accuracy of the measurements in this case is worse compared to the case where the pole is positioned vertically due to inaccurate compass readings. It also decreases with the increase of the inclination angle. It should be noted that the desired accuracy of the measured position (usually, 1-5 cm) imposes quite severe requirements on the desired accuracy of the attitude matrix since the error in the position is proportional to the angular error multiplied by the length of the pole. For instance, for a pole of (typical) length 2-2.5 m, an angular error 0.05 rad results in the position error about $10-12.5 \mathrm{~cm}$, which is usually unacceptable.

In the framework of the new approach, the surveyor places the bottom end of the pole to the point to be measured with the pole being inclined in an arbitrary direction and, instead of fixing the pole position, makes it move for a few seconds by arbitrarily changing the inclination angle. The position of the bottom end of the pole is determined then by processing the measurements of the gyros and the antenna velocity as described in the subsequent sections. Magnetic disturbances do not affect the result, since the compass is not used.

The paper is organized as follows. In Section 2, we set the problem to be discussed in the paper and formulate assumptions imposed on the equipment. A description of the proposed approach is presented in Section 3. The application of the proposed method for determining a point on the ground by using field data collected with the help of a receiver and an IMU installed on a geodetic pole is illustrated in Section 4. The results obtained show that the accuracy of determining the initial heading angle and, eventually, the position is better than that obtained with the help of a magnetometer. In Section 5, a modified version of the method is presented. It makes use of the accelerometer measurements to determine the initial pitch and roll angles, whereas the initial heading angle is found by solving an optimization problem of reduced size.

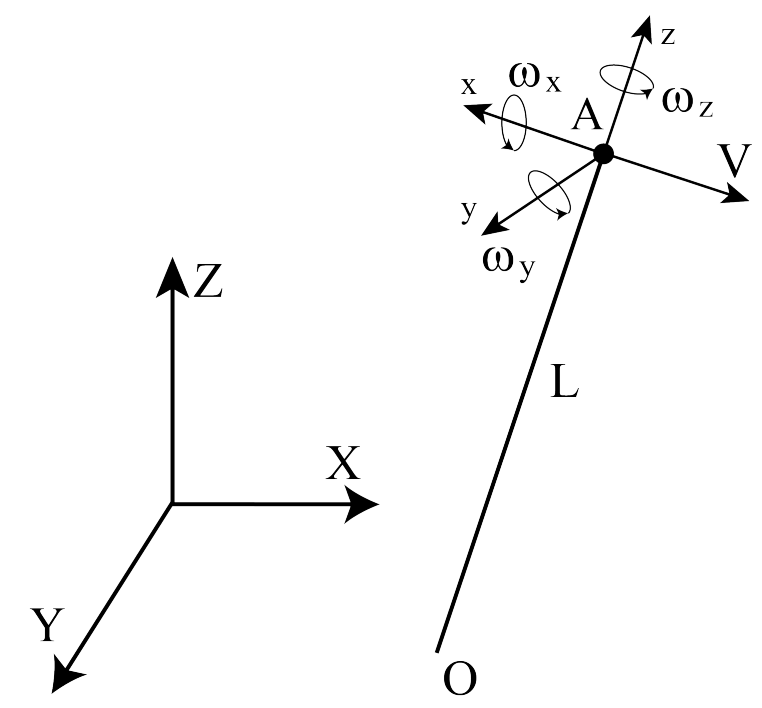

Figure 1: Rotating rigid body with fixed point $O$.

\section{Problem Statement}

We consider a rigid body (device) with a fixed point $O$ (e.g., like that in Figure 1) performing motion in a time interval $\left[t_{0}, t_{f}\right]$. Let $X, Y, Z$ denote the axes of an Earthrelated inertial frame and $x, y, z$ denote the axes of the body frame. Let the body be equipped with a satellite antenna installed at a point $A$, a GNSS receiver (further, simply a receiver), and an IMU. A typical IMU includes usually 3 gyroscopes (gyros), three accelerometers or an inclinometer (2-axis accelerometer), and a compass. However, the method presented below needs only the 3-axis gyro, which measures components of the angular velocity vector $\Omega=\left[\omega_{x}, \omega_{y}, \omega_{z}\right]^{\mathrm{T}}$ in the body frame. The receiver measures the coordinates of the antenna (point $A$ ) and its velocity vector $V^{m}$ in the inertial coordinate system. By $Q$, we denote the attitude matrix of the body, i.e., the matrix transforming the coordinates of a vector in the body frame into those in the inertial frame.

We assume that the gyro and receiver measurements are synchronized and that the rate of measurements is sufficiently high (100 Hz or higher), which allows us to consider time as a continuous variable. Suppose that the gyros have been calibrated immediately before measurements and that the measurement time $t_{f}-t_{0}$ is not too big, so that the error of the attitude determination due to unaccounted or varying biases of the gyros can be neglected. The goal is to determine the attitude matrix $Q(t) \forall t \in\left[t_{0}, t_{f}\right]$ based on the above-specified measurements. 


\section{Description of the Proposed Approach}

We will take advantage of the fact that the velocity of the antenna measured by means of the receiver can also be calculated from the known angular velocity of the body and its geometry if the attitude matrix is known. Indeed, if a point $O$ of the moving body is fixed, the velocity of the point $A$ is given by the formula [5] $V^{A}=\omega_{I} \times P_{I}=Q \Omega \times Q P_{B}$, where $\omega_{I}=Q \Omega$ is the angular velocity vector in the inertial frame, $P_{B}$ is the vector $\overrightarrow{O A}$ written in the body-frame coordinate system, and $P_{I}=Q P_{B}$ is the coordinate representation of this vector in the inertial frame.

For a given vector $a=\left[a_{1}, a_{2}, a_{3}\right]^{\mathrm{T}}$, we will use the notation $a^{\times}$to denote the skew-symmetric matrix associated with $a$ :

$$
a^{\times}=\left(\begin{array}{ccc}
0 & -a_{3} & a_{2} \\
a_{3} & 0 & -a_{1} \\
-a_{2} & a_{1} & 0
\end{array}\right),
$$

which allows us to represent the vector product of two vectors $a$ and $b$ as a matrix multiplication: $a \times b=a^{\times} b$. Using this formula, orthogonality of the attitude matrix $Q$, and the well-known formula $(Q a)^{\times}=Q a^{\times} Q^{\mathrm{T}}[6]$, we can write $V^{A}$ as

$$
V^{A}=(Q \Omega)^{\times} Q P_{B}=Q \Omega^{\times} Q^{\mathrm{T}} Q P_{B}=Q \Omega^{\times} P_{B},
$$

where

$$
\Omega^{\times}=\left(\begin{array}{ccc}
0 & -\omega_{z} & \omega_{y} \\
\omega_{z} & 0 & -\omega_{x} \\
-\omega_{y} & \omega_{x} & 0
\end{array}\right) .
$$

The matrix $Q$ is the solution of the Poisson kinematic equation [6]

$$
\dot{Q}=Q \Omega^{\times}, Q\left(t_{0}\right)=Q_{0} .
$$

Thus, the velocity of the point $A$ at any time is completely determined by the orientation of the body at the initial time $t_{0}: V^{A}=V^{A}\left(t, Q_{0}\right)$. The problem is that the initial orientation of the body is not a priori known and cannot be measured with the given set of sensors.

Consider the functional

$$
\Phi\left(Q_{0}\right)=\int_{t_{0}}^{t_{f}}\left(V^{A}\left(\tau, Q_{0}\right)-V^{m}(\tau)\right)^{\mathrm{T}}\left(V^{A}\left(\tau, Q_{0}\right)-V^{m}(\tau)\right) d \tau .
$$

It is clear that the global minimum of the functional is attained when $Q_{0}$ coincides with the true attitude matrix of the body at the initial moment. Hence, the desired initial attitude matrix can be found by minimizing functional (4) if the linear velocity of the antenna $V^{A}(t)$ is not identically equal to zero $\left(\Omega^{\times} P_{B} \neq 0\right)$.
Let us parameterize the attitude matrix by means of the three Euler angles,

$$
Q_{0}=H\left(\psi_{0}\right) T\left(\theta_{0}\right) R\left(\phi_{0}\right),
$$

where $H\left(\psi_{0}\right), T\left(\theta_{0}\right)$, and $R\left(\phi_{0}\right)$ are matrices of rotation around axes $z, y$, and $x$ through angles $\psi_{0}, \theta_{0}, \phi_{0}$, respectively:

$$
\begin{aligned}
H\left(\psi_{0}\right) & =\left(\begin{array}{ccc}
\cos \psi_{0} & \sin \psi_{0} & 0 \\
-\sin \psi_{0} & \cos \psi_{0} & 0 \\
0 & 0 & 1
\end{array}\right), \\
T\left(\theta_{0}\right) & =\left(\begin{array}{ccc}
\cos \theta_{0} & 0 & \sin \theta_{0} \\
0 & 1 & 0 \\
-\sin \theta_{0} & 0 & \cos \theta_{0}
\end{array}\right), \\
R\left(\phi_{0}\right) & =\left(\begin{array}{ccc}
1 & 0 & 0 \\
0 & \cos \phi_{0} & \sin \phi_{0} \\
0 & -\sin \phi_{0} & \cos \phi_{0}
\end{array}\right) .
\end{aligned}
$$

Then, functional (4) is a function of three variables: $\Phi\left(Q_{0}\right)=\Phi\left(\psi_{0}, \theta_{0}, \phi_{0}\right)$, and determining the initial orientation reduces to minimizing the function of three variables. In our numerical experiments, we used the Broyden-Fletcher-Goldfarb-Shanno (BFGS) algorithm [7] to find the minimum of (4). The values of the function $\Phi$ and its derivatives $\Phi_{\psi_{0}}, \Phi_{\theta_{0}}$, and $\Phi_{\phi_{0}}$ are found by solving jointly the Poisson kinematic equation (3) and the four differential equations

$$
\begin{aligned}
\frac{d}{d t} \Phi & =\left(V^{A}-V^{m}\right)^{\mathrm{T}}\left(V^{A}-V^{m}\right), \Phi\left(t_{0}\right)=0, \\
\frac{d}{d t} \Phi_{\psi_{0}} & =2\left(V^{A}-V^{m}\right)^{\mathrm{T}} \frac{\partial V^{A}}{\partial \psi_{0}}, \Phi_{\psi_{0}}\left(t_{0}\right)=0, \\
\frac{d}{d t} \Phi_{\theta_{0}} & =2\left(V^{A}-V^{m}\right)^{\mathrm{T}} \frac{\partial V^{A}}{\partial \theta_{0}}, \Phi_{\theta_{0}}\left(t_{0}\right)=0, \\
\frac{d}{d t} \Phi_{\phi_{0}} & =2\left(V^{A}-V^{m}\right)^{\mathrm{T}} \frac{\partial V^{A}}{\partial \phi_{0}}, \Phi_{\phi_{0}}\left(t_{0}\right)=0
\end{aligned}
$$

(i.e., the system of total differential order 13), where $\partial V^{A} / \partial \psi_{0}=\left(\partial Q_{0} / \partial \psi_{0}\right) Q_{0}^{\mathrm{T}} V^{A}, \partial V^{A} / \partial \theta_{0}=$ $\left(\partial Q_{0} / \partial \theta_{0}\right) Q_{0}^{\mathrm{T}} V^{A}, \partial V^{A} / \partial \phi_{0}=\left(\partial Q_{0} / \partial \phi_{0}\right) Q_{0}^{\mathrm{T}} V^{A}$; the derivatives of $Q_{0}$ are easily calculated by means of representation (5) as

$$
\begin{aligned}
& \frac{\partial Q_{0}}{\partial \psi_{0}}=H^{\prime}\left(\psi_{0}\right) T\left(\theta_{0}\right) R\left(\phi_{0}\right), \\
& \frac{\partial Q_{0}}{\partial \theta_{0}}=H\left(\psi_{0}\right) T^{\prime}\left(\theta_{0}\right) R\left(\phi_{0}\right), \\
& \frac{\partial Q_{0}}{\partial \phi_{0}}=H\left(\psi_{0}\right) T\left(\theta_{0}\right) R^{\prime}\left(\phi_{0}\right) ;
\end{aligned}
$$

and $H^{\prime}, T^{\prime}$, and $R^{\prime}$ are derivatives of the matrices $H\left(\psi_{0}\right), T\left(\theta_{0}\right)$, and $R\left(\phi_{0}\right)$, each of which is a function of one variable. 
The method described above allows one to get the attitude matrix of the device by postprocessing the measurements. However, it can easily be modified to get the attitude matrix in real time. Indeed, the true initial matrix $Q_{0}$ must ensure the global minimum to the functional for an arbitrary upper limit $t, t_{0}<t<t_{f}$, of the integral in (4). Then, one can use measurements in a shorter time interval $\left[t_{0}, t_{1}\right]$ to determine $Q_{0}$, to find the corresponding $Q_{1}=Q\left(t_{1}\right)$, and to calculate $Q(t)$ in the interval $\left[t_{1}, t_{f}\right]$ in real time by solving the Poisson kinematic equation (3) with the initial matrix $Q_{1}$. Other modifications of the implementation of the method are also possible. For instance, the minimization problem can permanently be solved in a background mode, and its results can be used to update the solutions of the Poisson kinematic equation at fixed discrete time instants.

\section{Numerical Example}

As an illustration, we consider the application of the proposed method for determining GPS position of a point on the ground by means of a geodetic pole. In our field experiments, we used a standard pole of length 2.10 m equipped with the Javad Triumph $1 \mathrm{M}$ receiver with a built-in antenna [8]. The axes $X$ and $Y$ of the Earth-related inertial frame lie in the plane of the local horizon and are directed to the east and north, respectively, and the $Z$ axis is directed vertically upwards.

In the course of the experiments, the surveyor put the bottom end of the pole at a known location on the ground (to be able to compare it with the position determined by the proposed method), taking no care of whether the pole is vertical or inclined, and tilted the pole for a few seconds in different directions making the antenna move, while the bottom end remained fixed. The velocity of the antenna measured by the receiver and the gyroscope readings were recorded. The initial approximations for the angles $\theta_{0}$ and $\phi_{0}$ were taken to be zero (as if the pole stood vertically), $\theta_{0}^{0}=\phi_{0}^{0}=0$, and the initial approximation $\psi_{0}^{0}$ of the heading (yaw) angle $\psi_{0}$ was chosen arbitrarily. Our experiments showed that such a choice of the initial approximation almost always results in finding the global minimum of the functional. In practice, the desired initial angles can be very well approximated with the help of the compass and accelerometers, which are routinely available in any IMU, which will certainly facilitate the solution of the optimization problem.

The true initial angles $\psi_{0}, \theta_{0}$, and $\phi_{0}$ (and, thereby the matrix $Q_{0}$ ) were determined by minimizing functional (4).

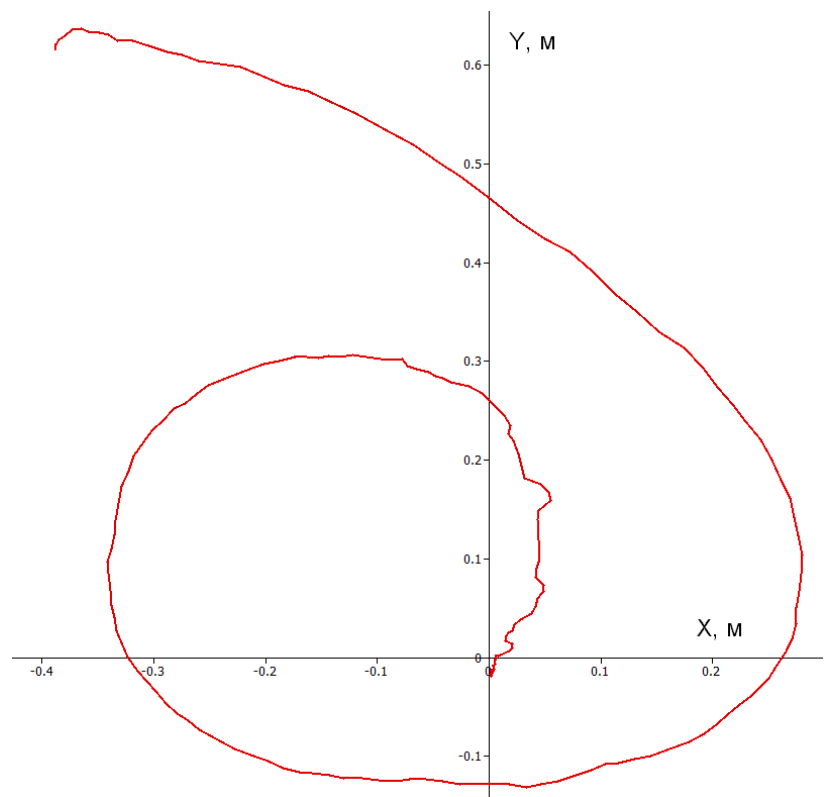

Figure 2: Projection of the antenna trajectory on the plane of local horizon.

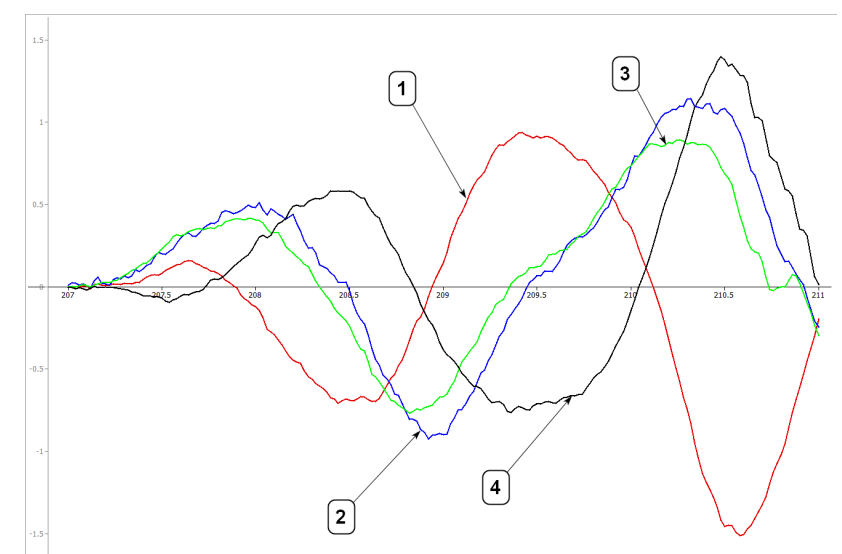

Figure 3: Comparison of the projections of the measured and calculated velocity of the antenna on the plane of local horizon $V_{X}^{m}(1)$, $V_{Y}^{m}$ (2) and $V_{X}^{A}$ (3), $V_{Y}^{A}$ (4) before the minimization of the functional.

Figures 2-4 show results of one of the field experiments demonstrating the application of the proposed method. Rotational motion was performed for 7 seconds around the bottom end of the pole, which was located on the ground at the origin of the inertial frame. The projection of the antenna trajectory on the plane of the local horizon is depicted in Figure 2. Figures 3 and 4 show components $V_{X}^{m}$ and $V_{Y}^{m}$ (curves marked by 1 and 2) of the measured velocity of the antenna (the same curves in both figures) and the components $V_{X}^{A}$ and $V_{Y}^{A}$ (curves 3 and 4) of the velocity $V^{A}$. These are calculated by formula (1) with the attitude matrix $Q(t)$ obtained by solving the Poisson kinematic equation (3) with different initial matrices $Q\left(t_{0}\right)$. The 


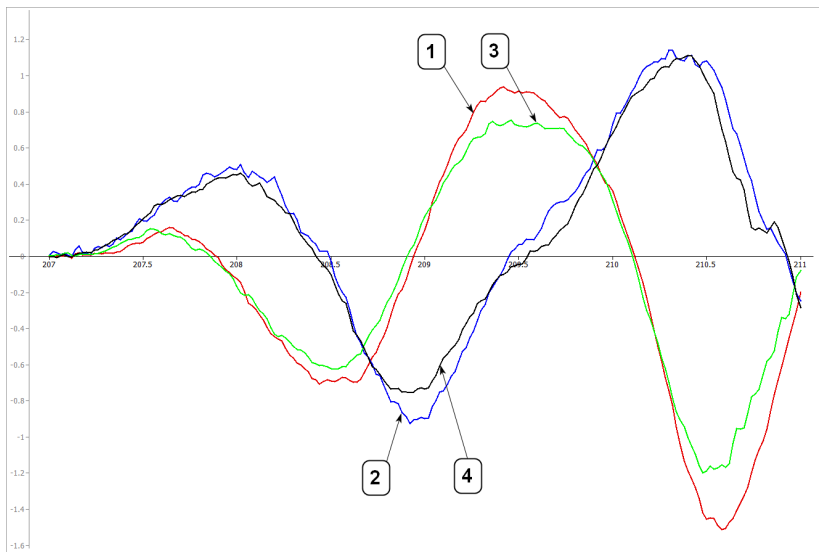

Figure 4: Comparison of the projections of the measured and calculated velocity of the antenna on the plane of local horizon $V_{X}^{m}(1)$, $V_{Y}^{m}$ (2) and $V_{X}^{A}$ (3), $V_{Y}^{A}$ (4) after the minimization of the functional.

$Z$-components of the velocities are small and, hence, less informative and, therefore, are not presented. The velocity $V^{A}$ in Figure 3 was obtained for the initial matrix $Q\left(t_{0}\right)=I$ $\left(\psi_{0}^{0}=\theta_{0}^{0}=\phi_{0}^{0}=0\right)$. The corresponding value of the functional is $\Phi(I)=4.05$. As it can be seen, the calculated velocity $V^{A}$ differs considerably from the measured velocity $V^{m}$, since the initial attitude matrix is far from the true one.

By solving the minimization problem, it was found that the minimum of the functional was attained at $\psi_{0}=$ -1.342, $\theta_{0}=0.045, \phi_{0}=-0.007$ radians, with $\Phi\left(Q_{0}\right)=$ 0.129 . The horizontal components of the velocity $V^{A}$ corresponding to the attitude matrix found are shown in Figure 4. To get an idea of the accuracy of the attitude determination, the matrix obtained was used to determine the position of the bottom (fixed) end $O$ of the pole

$$
O_{\text {meas }}=P^{A}(t)-Q(t) P_{B},
$$

where $P^{A}(t)$ is the position of the antenna measured by the receiver, which was compared with the true position (the origin in our case). The latter was obtained by a conventional method using measurements collected when the pole was fixed in the vertical position. In the experiment described, the mean value of the position found was $[4.1,-6.7,0.5] \mathrm{cm}$. Numerous similar experiments showed that the deviations of the calculated positions from the true ones never exceeded $10 \mathrm{~cm}$, which implies that the r.m.s. error of angle determination was less than 0.02 radians.

\section{A Modified Version of the Method Employing Accelerometer Measurements}

As discussed above, finding the attitude matrix reduces basically to determining initial angles $\psi_{0}=\psi\left(t_{0}\right), \theta_{0}=\theta\left(t_{0}\right)$, and $\phi_{0}=\phi\left(t_{0}\right)$. The angles are found by minimizing functional (4), which is a function of three variables. Now, suppose that the IMU installed on the body also includes accelerometers and that the body was at rest at $t=t_{0}$. The latter means that, at the initial moment, the accelerometers measure the vector of gravity in the body frame, and the initial pitch and roll angles $\theta_{0}$ and $\phi_{0}$ can be determined by the accelerometer readings $a_{x}^{0}=a_{x}\left(t_{0}\right), a_{y}^{0}=a_{y}\left(t_{0}\right)$, and $a_{z}^{0}=a_{z}\left(t_{0}\right)$ as

$$
\phi_{0}=-\arcsin \frac{a_{y}^{0}}{a_{z}^{0}}, \theta_{0}=-\arcsin \frac{a_{x}^{0}}{a_{y}^{0} \sin \phi_{0}+a_{z}^{0} \cos \phi_{0}} .
$$

With $\theta_{0}$ and $\phi_{0}$ being fixed, the calculated velocity $V^{A}$ depends only on the heading angle $\psi_{0}, V^{A}=V^{A}\left(t, \psi_{0}\right)$, which allows us to simplify the task of determining the initial attitude matrix reducing it to the minimization of the function of one variable:

$\Phi\left(\psi_{0}\right)=\int_{t_{0}}^{t_{f}}\left(V^{A}\left(\tau, \psi_{0}\right)-V^{m}(\tau)\right)^{\mathrm{T}}\left(V^{A}\left(\tau, \psi_{0}\right)-V^{m}(\tau)\right) d \tau$.

Like in the basic version of the method, the values of the function $\Phi\left(\psi_{0}\right)$ and its derivative $\Phi_{\psi_{0}}\left(\psi_{0}\right)$ for a particular $\psi_{0}$ are found by solving the following system of 11 differential equations (two equations less than in the basic version):

$$
\begin{aligned}
\frac{d}{d t} \Phi & =\left(Q \Omega^{\times} P_{B}-V^{m}\right)^{\mathrm{T}}\left(Q \Omega^{\times} P_{B}-V^{m}\right), \\
\frac{d}{d t} \Phi_{\psi_{0}} & =2\left(Q \Omega^{\times} P_{B}-V^{m}\right)^{\mathrm{T}} H^{\prime}\left(\psi_{0}\right) H^{\mathrm{T}}\left(\psi_{0}\right) Q \Omega^{\times} P_{B}, \\
\frac{d}{d t} Q & =Q \Omega^{\times}
\end{aligned}
$$

with the initial values $\Phi\left(t_{0}\right)=0, \Phi_{\psi_{0}}\left(t_{0}\right)=0$, and $Q\left(t_{0}\right)=$ $Q_{0}\left(\psi_{0}\right)$, respectively.

The modified version of the method was applied to the data collected in the field experiment described in the previous section (see Figure 2). The initial roll and pitch angles found from the accelerometer readings were $\phi_{0}=$ 0.008 and $\theta_{0}=-0.009$. The solution of the minimization problem yielded $\psi_{0}=-1.345$ and $\Phi\left(\psi_{0}\right)=0.132$ (cf. with 0.129 obtained by the basic method). As can be seen, the minimum of the functional $\Phi\left(\psi_{0}\right)$ only slightly differs from the minimum of functional (4), which means that 
the calculated velocities $V^{A}$ in both cases are very close to each other, so that their plots are almost indistinguishable. Hence, the plots of the measured and calculated velocities look the same as those in Figure 4 and, therefore, are not presented. Although the value of the functional obtained by the new method is a little bit greater than that obtained by the basic method, the deviation of the calculated position of the pole bottom end from the true position turned out less than that in the previous case. The averaged coordinates of the estimated position $O_{\text {meas }}$ calculated by formula (6) were equal to $[2.2,3.0,0.5] \mathrm{cm}$.

Here, it should be noted that, in the general case, the accuracy of the modified method can be both better and worse than the accuracy of the basic method, which depends on the quality of the accelerometers employed in the IMU. On the one hand, the minimum of the functional $\Phi\left(\psi_{0}\right)$ is evidently not less than the minimum of the functional (4). On the other hand, due to unaccounted gyro biases (which always exist even if the gyros have been calibrated) and errors in the gyro scale factors, the minimum of the functional (4) is not necessarily achieved at the true initial attitude matrix. Then, if the accelerometers are accurate enough and are well calibrated, it may happen that the approximations of the initial roll and pitch angles obtained by means of the accelerometers are more accurate than those obtained by minimizing the functional. It is this case that was observed in the experiments described in this and previous sections.

\section{Conclusions}

In the paper, the problem of determining attitude was discussed. A new method for finding the attitude matrix for a moving rigid body with a fixed point has been proposed. The base method relies on measurements of the angular and linear velocities and does not use a compass and accelerometers. The method reduces the problem of determining the attitude to solving a minimization problem for a function of three variables. A modified version of the method has also been proposed for a particular case of the problem under consideration that makes use of accelerometers at the initial time moment. The use of the accelerometers reduces the complexity of the problem to be solved and improves the accuracy of determining the attitude if the accelerometers are accurate enough. The discussion has been illustrated by results of field experiments.

The proposed method can be employed in precise geodetic measurements carried out with the use of a geodetic pole with a satellite antenna installed on its top when, for some reasons, the surveyor cannot position the pole vertically (e.g., near walls or buildings). To recalculate the measured position of the antenna to the desired position of the pole bottom end, the attitude matrix is needed, which can be determined by the method discussed in this paper. The use of the traditional approach, which relies on a compass and accelerometers, in this case does not ensure the desired accuracy of determining the attitude due to magnetic disturbances (both external ones and those induced by the receiver) affecting the compass readings badly.

Acknowledgement: This work was partially supported by Basic Research Program I.7 "New Developments in Perspective Areas of Energetics, Mechanics and Robotics" of the Presidium of Russian Academy of Sciences and by the Russian Foundation for Basic Research, project no. 18-0800531.

\section{References}

[1] Gebre-Egziabher, D., Elkaimy, G.H., Powell, J.D., Parkinson, B.W., Calibration of strapdown magnetometers in the magnetic field domain, Journal of Aerospace Engineering, 2006, 19(2), 87-102.

[2] Ozyagcilar, T., Implementing a tilt-compensated eCompass using accelerometer and magnetometer sensors, Freescale Semiconductor, Application Note, AN4248, Rev. 3, 2012.

[3] Pesterev, A.V., Morozov, Yu.V., Matrosov, I.V., Ashjaee, J., Estimation of the magnetic field generated by UAV in flight, Proceedings of the 25th Saint Petersburg International Conference on Integrated Navigation Systems (2018, Saint Petersburg, Russia), 2018, 1-4, DOI: 10.23919/ICINS.2018.8405882

[4] Roberts, A., Tayebi, A., On the attitude estimation of accelerating rigid bodies using GPS and IMU measurements, Proceedings of the 50th IEEE Conference on Decision and Control and European Control Conference (CDC-ECC), 2011, 8088-8093.

[5] Aizerman, M.A., Classical Mechanics, Nauka, Moscow, 1974 (in Russian).

[6] Amel'kin, N.I., Kinematics and Dynamics of Rigid Bodies, Izdatel'stvo MFTI, Moscow, 2000 (in Russian).

[7] Fletcher, R., Practical Methods of Optimization, Wiley, New York, 1987.

[8] www.javad.com 